Prix Nobel de Chimie 2010

Richard F. Heck, દi-ichi Negishi, Akira Suzuki

\title{
Le palladium, un catalyseur magique au service des chimistes
}

> Le prix Nobel de chimie 2010 a été attribué à trois chercheurs: Richard F. Heck, Professeur émérite de l'Université du Delaware (États-Unis), Ei-ichi Negishi, Professeur émérite de chimie organique à l'Université de Purdue (États-Unis) et Akira Suzuki, Professeur émérite à I'Université d'Hokkaido (Japon) pour leurs travaux pionniers

\section{Jean-Pierre Genet} dans la découverte et le rayonnement du «couplage croisé catalysé par le palladium et ses applications en synthèse organique ». Ces travaux ont radicalement révolutionné les méthodes de création de la liaison carbone-carbone. L'essor de ces outils offre des possibilités infinies pour le développement et la production de médicaments (anticancéreux, antibiotiques, hormones), de composés agrochimiques et de nouveaux matériaux organiques. <

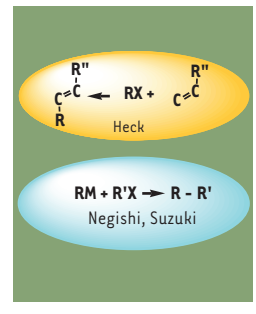

Professeur émérite, દ́cole nationale supérieure de chimie de Paris, Laboratoire Charles Friedel, UMR 7223, 11, rue Pierre et Marie Curie, 75231 Paris, France. jean-pierre-genet@chimie-paristech.fr

Les découvertes faites par les lauréats sont le fruit d'une maturation lente: les premiers modèles de procédés véritablement catalytiques apparaissent en 1975. Les travaux des Nobel 2010 concernent le «couplage croisé », c'est-à-dire la substitution d'un halogénure d'aryle, vinyle ou alkyle par un nucléophile organomé-

Le palladium, découvert en 1903 par Wollatson, est un métal rare. Le nom qui lui fut attribué l'année suivant sa découverte dérive de l'astéroïde Pallas, Palladium en grec. Cette appellation fait référence à la déesse mythologique de la sagesse et du savoir. Le palladium est utilisé dans l'industrie automobile (pots catalytiques), dans la fabrication des composants électroniques pour les portables, les ordinateurs et l'électronique. L'activité du palladium et de ses dérivés organométalliques synthétiquement utiles est favorisée principalement aux états d'oxydation 0 et 2. La chimie du palladium est très «chimiosélective » et a magistralement complété la traditionnelle chimie des Grignards ${ }^{1}$ et des organolithiens ${ }^{2}$. Le développement des réactions catalytiques utilisant le palladium en synthèse organique a été beaucoup moins rapide que celui de la chimie organométallique classique.

${ }^{1}$ Du nom de Victor Grignard, prix Nobel à 41 ans, qui fait partie des grands chimistes français qui ont laissé leur nom dans l'histoire. C'est en effet le seul qui figure dans tous les ouvrages de chimie organique, du plus simple au plus complexe, pour avoir mis au point la préparation des organomagnésiens, une classe de molécules particulièrement utilisées en synthèse organique.

${ }^{2}$ Les organomagnésiens et les organolithiens font partie de la famille des organométalliques définis comme des composés dans lesquels il existe une liaison métal-carbone. (Figure 1). Ces réactions procèdent selon les étapes élémentaires de la chimie organométallique : addition oxydante, transmétallation et élimination réductrice. L'utilisation significative de ces méthodes a connu un véritable essor vers le milieu des années 1980. La sélection du catalyseur pour une réaction donnée nécessite un ajustement fin des propriétés électroniques et stériques du ligand ( $L$ ), partenaire associé au palladium. Le palladium est relativement peu toxique et certains complexes du palladium peuvent être employés à l'air, sous forme supportée $e^{3}$, en milieu liquide ionique ou dans l'eau. C'est un solvant idéal qui permet en particulier le recyclage du catalyseur coûteux. Ces aspects sont particulièrement importants dans le monde industriel et contribuent à une chimie respectueuse de l'environnement.

\section{Le Professeur Richard F. Heck}

Né en 1931, il obtient son degré de bachelor à l'Université de Californie (UCLA) en 1952 puis il soutient son Docto-

${ }^{3}$ Ce sont des catalyseurs au palladium fixés sur des supports solides que l'on peut donc facilement séparer du milieu réactionnel et donc des composés synthétisés. 


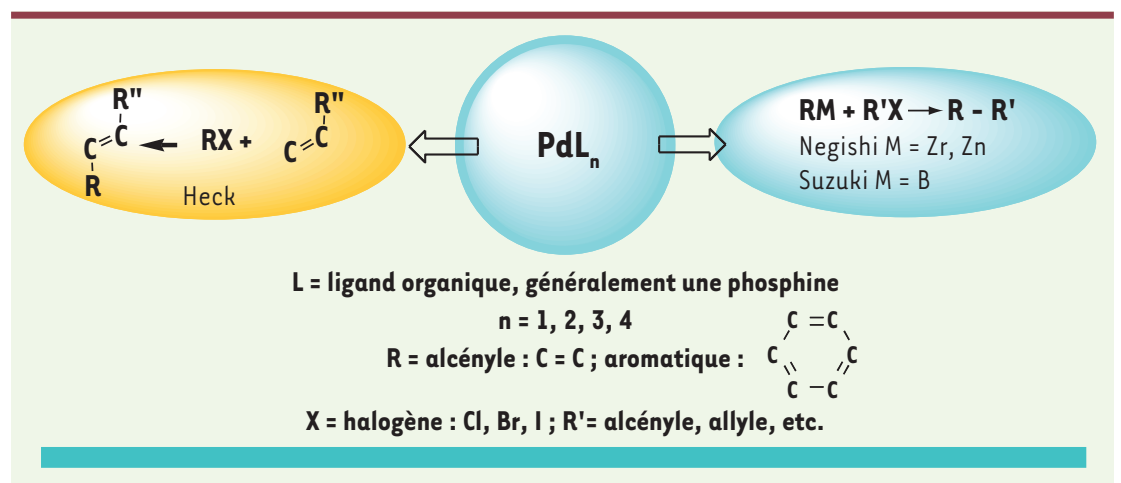

Figure 1. Couplage croisé.

de partir aux États-Unis, à l'Université de Pennsylvanie, pour y effectuer une thèse en chimie organique centrée sur les polymères et soutenue en 1963. II rejoint ensuite le Professeur H.C. Brown à l'Université de Purdue pour un stage post-doctoral puis est nommé assistant Professor à l'Université de Syracuse et full Professor à Purdue en 1979. II devient en 1999 H.C. Brown distinguished

rat en 1954. R.F. Heck effectue ensuite 2 années de stage post-doctoral à I'ETH à Zurich avant de rejoindre la société Hercules, Wilmington, Delaware. Ses remarquables recherches menées au sein de l'entreprise le conduisent à être recruté en 1971 par l'Université de Delaware dans le Département de chimie et biochimie. Retraité en 1989, il est nommé depuis Professeur émérite «Wallis F. Harrington». En 1968 R. F. Heck publie une série d'articles dans lesquels une liaison carbone-carbone est créée par catalyse avec le palladium [1]. II s'agissait d'une réelle avancée dans le domaine de la catalyse. Cependant, cette réaction présentait des inconvénients majeurs nécessitant un équivalent de palladium et l'utilisation de dérivés aromatiques contenant du mercure ou de l'étain, composés particulièrement toxiques. Au début des années 1970, R. F. Heck [2] et T. Mizoroki (Japon) [3] décrivent indépendamment pour la première fois la réaction de couplage direct d'oléfines avec des aryles et alcényles halogénés en présence de palladium catalytique. L'essor de cette efficace formation de liaison C-C dite de Mizoroki-Heck débute seulement vers la deuxième moitié des années 1980 (Figure 1) [4]. Elle est fiable, possède une grande flexibilité, est compatible avec de nombreux groupes fonctionnels et repose sur la facilité de préparation des substrats de départ. Les versions intramoléculaires et énantiosélectives ont été également développées très récemment, permettant de créer des centres stéréogènes quaternaires ouvrant des voies d'accès aux composés carbocycliques et hétérocycliques [5].

La réaction de Mizoroki-Heck, devenue immensément populaire, est indispensable en synthèse organique. Elle permet l'accès à de nombreuses molécules d'intérêt thérapeutique (Figure 2): les anticancéreux dont le Taxol ${ }^{\circledR}$; le GI147211C (Glaxo), un analogue de la camptothécine, un inhibiteur de la topo-isomérase de type I; les hormones (estrone); la 1 1 25-dihydroxyvitamine D3; des analgésiques (morphine), la codéine, la capnellène, composé polycyclique anti-douleur.

La synthèse industrielle du Naproxène ${ }^{\circledR}$, anti-inflammatoire non stéroïdien, à l'aide de la réaction de Heck, a été developpée. L'intermédiaire-clé du Singulair (Merck), un médicament anti-asthmatique et l'herbicide Prosulfuron ${ }^{\circledR}$ sont produits par condensation de MizorokiHeck à une échelle supérieure, atteignant une tonne par année.

\section{Le Professeur Ei-ichi Negishi}

Il obtient en 1958 le degré de bachelor à I'Université de Tokyo puis il travaille ensuite deux années au sein de la société Teijin au Japon avant
Professor. Ei-ichi Negishi a publié plus de 400 articles ou chapitres de livres. Il a écrit deux livres dont Handbook of organopalladium chemistry for organic synthesis, en 2002. Dès 1977, il publie une série d'articles importants décrivant des réactions de couplage au palladium entre un organométallique contenant du zinc, de l'aluminium ou du zirconium $[6,7]$ et un dérivé halogéné organique. La découverte et le développement de réactions catalytiques ont permis l'élaboration de liaisons carbone-carbone dans des édifices moléculaires complexes $[8,9]$. Ces réactions de couplage permettent de synthétiser économiquement et efficacement une grande variété d'oléfines di- et tri-substituées avec un contrôle parfait de la stéréosélectivité [10].

Ces réactions qui portent le nom de «couplage de Negishi » sont devenues des outils efficaces pour la synthèse de composés biologiquement actifs (Figure 2) : vitamine A; carbacycline, un analogue de la prostacycline $A$; prostaglandines; grandisol $^{4}$; nakienone $A$ et $B$; lissoclinolide $B$; carotène; xéruline, mokupalide. Ces méthodes de synthèse ont été rapidement adoptées par plus de 60 laboratoires réputés dans le domaine de la synthèse totale de produits naturels d'intérêt biologique. Par exemple: le discodermolide, un puissant stabilisateur du fuseau ayant une activité cytotoxique comparable à celle des taxoïdes; l'acide zaragocique (squalene synthase inhibitor) ; l'eunicénone A ; la callystatine, un cytotoxique; l'acide xérulinique, au fort potentiel hypocholestérolémiant; salicylihalamides $A$ et $B$, des antitumoraux qui inhibent les ATPases vacuolaires $H_{+}$; l'épothilone, une molécule cytotoxique de type taxane ciblant les microtubules; la murisoline, un inhiteur du complexe mitochondrial I.

La préparation à grande échelle de composés pharmaceutiques tels que le PDE472 (Novartis), un inhibiteur de la phosphodiestérase; le 2-chloro(-pyridin-2-yl) pyrimidine, un intermédiaire-clé dans la synthèse d'inhibiteur sélectif du PDE-V (phosphodiestérase), un puissant vasodilatateur (Johnson \& Johnson), utilise cette méthodologie.

${ }^{4}$ Le grandisol est un composant d'une phéromone sexuelle mâle d'un charançon de la graine du coton qui a causé des pertes importantes dans les récoltes aux États-Unis. La synthèse de cette phéromone a été réalisée en 1970. 


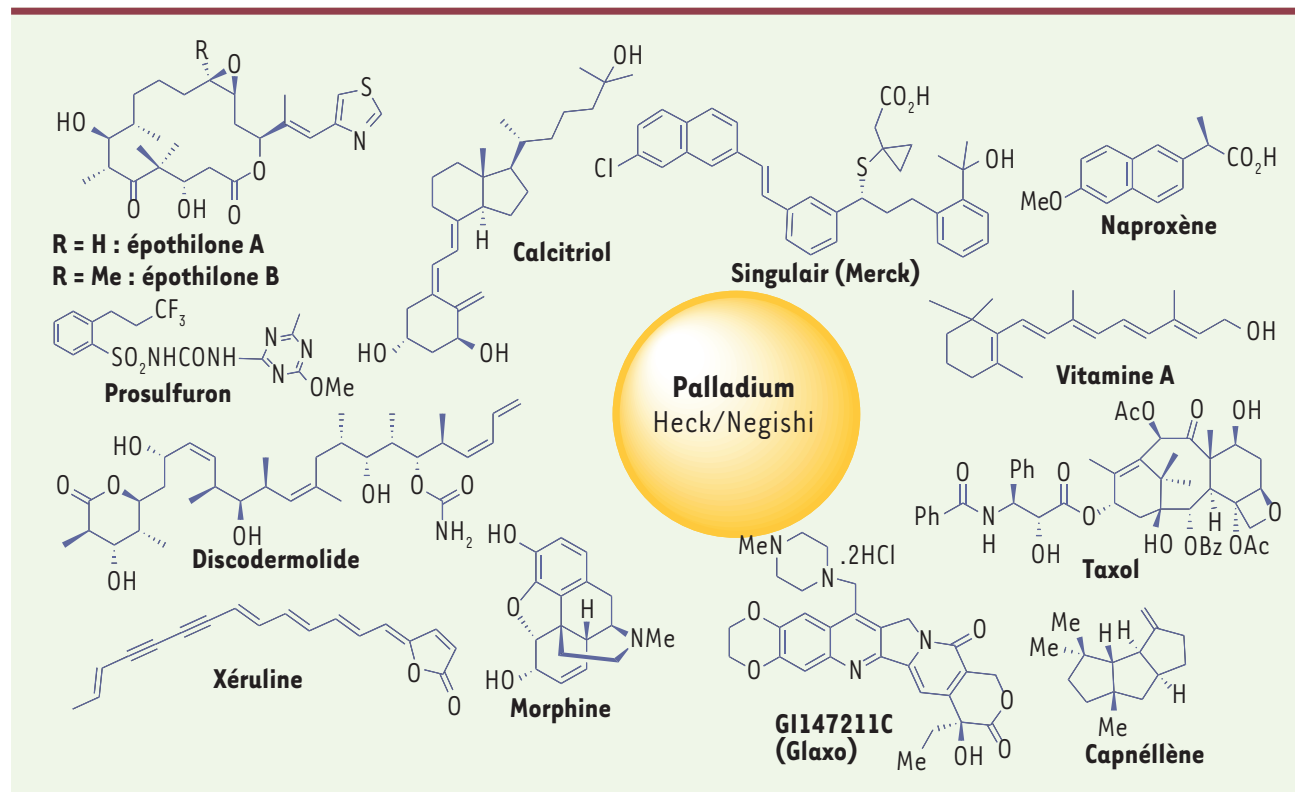

Figure 2. Composés d'intérêt biologique synthétisés à l'aide des méthodologies Heck et Negishi.

\section{Le Professur Akira Suzuki}

Né le 12 septembre 1930 à Mukawa-cho, Hokkaido, A. Suzuki a obtenu son doctorat à l'Université d'Hokkaido en 1959. II est nommé assistant Professor dans le département de cette université en 1961. II effectue un stage dans le laboratoire de H.C. Brown à l'Université de Purdue (1963 à 1965). II devient ensuite Professeur dans le Département de chimie appliquée à l'Université d'Hokkaido jusqu'à sa retraite en 1994. II rejoint ensuite successivement les Universités d'Okayama et de Kurashiki poursuivant ses recherches dans le domaine de la chimie du bore.

Les premières méthodes de couplages croisés catalysés par les complexes du palladium décrites par Suzuki et Miyaura concernent la réaction d'alcényles boranes avec des dérivés halogénés vinyliques de stéréochimie définie [11, 12]. Ces auteurs ont également décrit en 1981 la première méthode générale de synthèse de dérivés biaryliques, par couplage croisé entre un dérivé arylhalogénure et un composé arylboronique en présence de (tétrakis)triphénylphosphine palladium: $\mathrm{Pd}\left(\mathrm{PPh}_{3}\right)_{4}$. À la suite de cette découverte, de nombreuses améliorations des conditions expérimentales ont été publiées [13] rendant cette réaction extrêmement efficace et utile pour la synthèse de composés biologiquement actifs.

La méthode est complémentaire du couplage de Negishi et extrêmement efficace car elle tolère la présence de groupes fonctionnels sur les partenaires participant au couplage. II s'agit d'un progrès considérable par rapport aux anciennes méthodes de synthèse nécessitant des réactifs incompatibles avec des molécules possédant des groupes polaires; de plus le bore n'est pas toxique. Les motifs de type biphényle sont présents dans de nombreuses molécules bioactives. La méthodologie de Suzuki-Miyaura est également utile pour la recherche, en chimie médicinale, de nouveaux principes actifs à l'aide des deux librairies d'acides boroniques d'une part et de dérivés halogénés d'autre part. Le couplage palladocatalysé ouvre la voie à la découverte de structures inédites.
Un nombre considérable de composés biologiquement actifs possédant des structures complexes [14] ont été synthétisés grâce à cette technologie (Figure 3) dont certains sont commercialisés. Citons en quelques-uns: la palytoxine, une neurotoxine issue des algues toxiques et qui agit sur la pompe $\mathrm{Na}^{+} / \mathrm{K}^{+}$-ATPase ; le losartan ou le valsartan (Novartis), antagonistes du récepteur de l'angiotensine II utilisés dans le traitement de l'hypertension; les carbapénèmes, antibiotiques de la famille des bêta-lactamines; le myxalamide, antibiotique polyène; l'épothilone, molécule cytotoxique inhibitrice des microtubules; la vancomycine, un antibiotique de la famille des glycopeptides ; le Cp-724714, inhibiteur du récepteur HER-2 à activité tyrosine kinase, en cours d'essai clinique; ABT-963, inhibiteur de Cox-2; la prostaglandine $\mathrm{PGF}_{2}$; GSK 1360707, inhibiteur de la recapture des neurotransmetteurs; ou certains fongicides comme le boscalide (BASF).

\section{Conclusion et perspectives}

Cette excellente méthodologie était cependant limitée aux dérivés halogénés bromés iodés et aux pseudo halogénures. La modification du catalyseur de palladium par introduction de nouveaux ligands tels que les phosphines riches en électrons à permis récemment d'étendre cette réaction à des dérivés halogénés chlorés variés moins coûteux [15]. De nouvelles voies d'accès aux acides boroniques et boronates d'aryles ont été découvertes par une remarquable activation de liaison carbone-hydrogène à l'aide de complexes organométalliques dérivés de l'iridium [16]. Cette méthode, couplée avec la réaction de Suzuki-Miyaura, a permis très récemment d'effectuer une synthèse élégante du complanamide. 


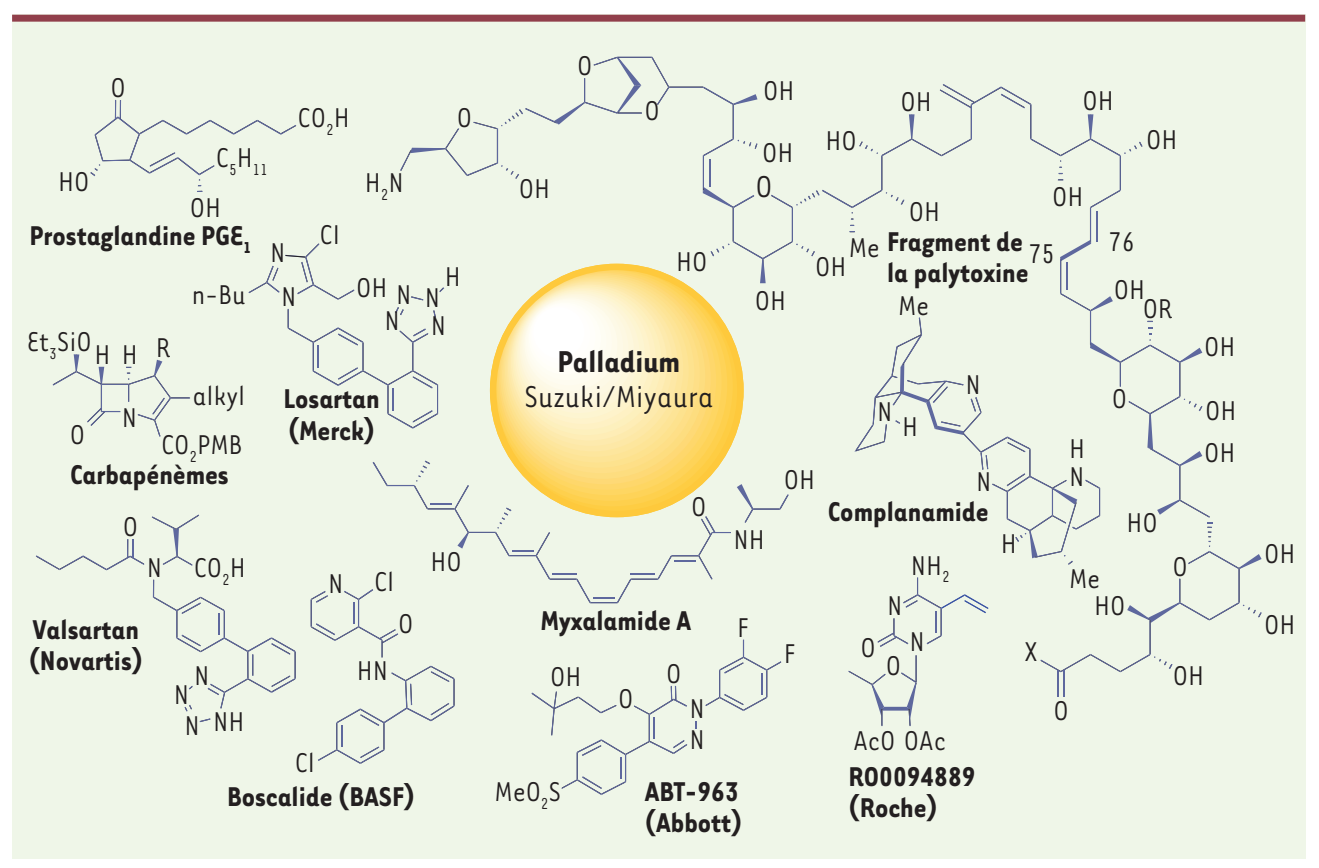

Figure 3. Composés biologiquement actifs obtenus par couplage de Suzuki-Miyaura.

La méthode extrêmement populaire de Suzuki-Miyaura est basée principalement sur l'utilisation des acides ou esters boroniques. Certains de ces dérivés manquent parfois de stabilité. Une solution à ce problème a été apportée récemment par l'utilisation de composés du bore, plus stables, les organotrifluoroborates de potassium [17]. Ces derniers ont conduit à de nouveaux développements du couplage de Suzuki-Miyaura au palladium. Ainsi l'utilisation du vinyltrifluoroborate de potassium a permis à Hoffman-la Roche d'effectuer la synthèse à grande échelle d'un anticancéreux, le R00094889, prodrogue convertie en un inhibiteur de la dihydropyrimidine déhydrogènase dans les tumeurs.

Les travaux de R.F. Heck, E.I. Negishi et A. Suzuki ont contribué à l'essor de la synthèse organique moderne, permettant de contrôler avec efficacité la réactivité et la sélectivité. Ces méthodes, et celles développées par Sonogashira et Tsuji-Trost, couplages également catalysés par le palladium [18], contribuent à une chimie plus respectueuse de l'environnement. Ces méthodes catalytiques permettent de réduire de façon spectaculaire le nombre d'étapes d'une synthèse totale. De nouvelles découvertes sont attendues et contribueront sans aucun doute à élaborer de façon encore plus expéditive des molécules biologiquement actives complexes. $\diamond$

The palladium, a magic catalyst for the chemists

\section{CONFLIT D'INTÉRÊTS}

L'auteur déclare n'avoir aucun conflit d'intérêts concernant les données publiées dans cet article.

\section{RÉFÉRENCES}

1. Heck RF. Acylation, methylation, and carboxyalkylation of olefins by Group VIII metal derivatives. J Am Chem Soc 1968; 90 : 5518-26.

2. Heck RF, Nolley JP. Palladium-catalyzed vinylic hydrogen substitution reactions with aryl, benzyl, and styryl halides. J Org Chem Soc 1972; $37: 2320-2$.

3. Mizoroki T, Mori K, Ozaki O. Arylation of olefin with iodobenzene catalyzed by Palladium. Bull Chem Soc Jpn $1973 ; 46: 1505-8$.
4. Heck RF. Palladium-catalyzed reactions of organic halides with olefins. Acc Chem Res 1979; $12:$ 146-51.

5. Brase S, De Meijere A, Dyker G, et al. Handbook of organopalladium chemistry for organic synthesis. Chapters IV, IV.1, IV1.2, IV.2.2., IV.2. In : Negishi $\varepsilon$, ed. New York: John Wiley and Sons Inc, 2002: 1123-315.

6. Negishi $\varepsilon$, King A0, Okukado N. Selective carbon-carbon bond formation via transition metal catalysis. A highly selective synthesis of unsymmetrical biaryls and diarylmethanes by the Nickel-or Palladium-catalyzed reaction of aryl-and benzylzinc derivatives with aryl halides. J Org Chem 1977 ; 42 : 1821-3.

7. Negishi $\varepsilon$. Controlled carbometalation as a new tool for carbon-carbon bond formation and its application to cyclization. Acc Chem Res $1987 ; 20: 65-72$.

8. Negishi $\varepsilon$, Hu $Q$, Huang Z, et al. Pd-Catalyzed alkenylation by the Negishi coupling. Aldrichimica Acta $2005 ; 38: 71-86$.

9. Negishi $\varepsilon$, Anastasia L. Palladium-catalyzed alkynylation. Chem Rev 2003; 103 : 1979-2017.

10. Negishi, $\varepsilon$, Wang Rao Xu Z. Alkyne elementometalation-Pd-catalyzed crosscoupling. Toward synthesis of all conceivable types of acyclic alkenes in high yields, efficiently, selectively, economically, and safely: green way. J Org Chem $2010 ; 75: 3151-82$.

11. Miyaura N, Yamada K, Suzuki A. A new stereospecific cross-coupling by the Palladium-catalyzed reaction of l-alkenylboranes with l-alkenyl or I-alkynyls halides. Tetrahedron Letters $1979 ; 36: 3437-40$.

12. Miyaura N, Yamada K, Suginome H, Suzuki A. Novel and convenient method for the stereo- and regiospecific synthesis of conjugated alkadienes and alkenynes via the Palladium-catalyzed cross-coupling reaction of 1-alkenylboranes with bromoalkenes and bromoalkynes. J Am Chem Soc 1985 ; 107 : 972-80.

13. Miyaura N, Suzuki A. Palladium-catalyzed cross-coupling reactions of organoboron compounds. Chem Rev $1995 ; 95: 2457-83$.

14. Suzuki A. Overview of the Suzuki protocol with Boron. Handbook of organopalladium chemistry for organic synthesis. Chap. III.2.2. In : Negishi $\varepsilon$, ed. New York: John Wiley and Sons Inc, 2002 : 249-62.

15. Buchwald SL (ed). Cross-coupling. Acc Chem Res 2008 ; 41 (nº spécial) : 1439-564. 16. Ishiyama T, Takagi J, Ishida K, et al. Mild iridium-catalyzed borylation of arenes. high turnover numbers, room temperature reactions, and isolation of a potential intermediate. J Am Chem Soc 2002 ; $124: 390-1$.

17. Darses S, Genet JP. Potassium organotrifluoroborates: new perspectives in organic synthesis. Chem Rev $2008 ; 108: 288-325$.

18. Nicolaou KC, Bulger PG, Sarlah D. Palladium-catalyzed cross-coupling reactions in total synthesis. Angew Chem Int Ed 2005 ; 44 : 4442-89.

TIRÉS À PART

J.P. Genet 